\title{
An Improved 40km Dual-Wavelength, Dual-Sagnac Sensor, with Enhanced Signal Processing Allowing Real-Time Location of Multiple Time-Varying Strain Disturbances
}

\author{
S.J.Russell, K.R.C.Brady, J.P.Dakin
}

Optoelectronics Research Center, University of Southampton, Highfield, Hampshire, UK, SO17-1BJ

Tel +44 (0) 23 80594530, Fax +44 (0) 23 80593149, email sr@orc.soton.ac.uk, jpd@orc.soton.ac.uk

\begin{abstract}
Updated results using an improved $40 \mathrm{~km}$ Sagnac detection system are presented. Real-time simultaneous positioning of multiple disturbances has now been realized. The new system has a higher output ASE source, improved WDM's and enhanced signal processing.
\end{abstract}

\section{INTRODUCTION}

For many years the Sagnac ring interferometer has been used to locate the longitudinal position of time-varying disturbances acting at a non-central location on its sensing loop. We now present improved results using our novel dual-wavelength, dual-Sagnac architecture [1] and report the first real-time detection and processing of multiple disturbances and their simultaneous, independent location. Our system now has improved signal/noise, reduced optical cross-talk and more sophisticated signal processing compared to earlier work [1]. The response of the system has now been fully modeled, allowing correction for optical cross-talk effects and the two wavelength operation of the system.

The Sagnac interferometer is inherently balanced and, provided there is a stable environment, the counterpropagating light will always interfere constructively. The ability of this interferometer to locate the position of a time-varying disturbance relies on the counter-propagating nature of the light and the break in symmetry that the disturbance creates when perturbing the loop.

When a time-varying strain, $\varepsilon(t)$, acts on the optical fiber, at a distance $z$ from the sensor loop center, it perturbs the phase of the guided light, $\phi(t)$. Due to its non-central location, it phase-modulates the light travelling in one direction before light travelling in the other. This results in a net phase difference, $\Delta \phi(t)$, between the two returning counter-propagating wavetrains when they interfere at the output of the loop. It was shown that, assuming a small perturbation amplitude, the phase difference, $\Delta \phi(t)$ can be given by, (1)

$$
\Delta \phi(t) \propto \frac{2 z}{V_{g}} \frac{d \phi(t)}{d t}
$$

where $V_{g}$ is the group velocity of the guided light.

Unfortunately we have a sensor which returns two unknowns, i.e. the rate of change of the phase perturbation and its position on the loop. Dakin et al [2,3], was the first publication to present a solution to this problem. By interfering a fraction of the light that had traveled in one direction around the Sagnac loop with light from the source, delayed via a fiber loop of suitable length, to form a balanced-path fiber Mach-Zehnder. The output of this gave a direct measure of $d \phi / d t$, allowing the Sagnac response to be decoded and the position of the disturbance found.

More recently $[4,5,6,7,8]$, various architectures using twin Sagnac configurations have been suggested, avoiding the need for accurately balancing the optical paths. These reported experimental (laboratory) location of disturbances over sensor loop lengths of up to $800 \mathrm{~m}$. They operated with either twin-source (wavelength multiplexed) [5,8], or intrinsically lossy arrangements, using directional $3 \mathrm{~dB}$ couplers and twin detectors $[4,6,7]$. With the latter, the minimum theoretical loss of a dual-Sagnac system using $3 \mathrm{~dB}$ couplers is $18 \mathrm{~dB}$ in each Sagnac loop.

We shall now report on an update of our recently reported optical architecture [1], which incorporates the following novel improvements:

(a) An improved higher-power, short coherence-length, Erbium-doped, super-luminescent fiber source, now giving a maximum power of $4 \mathrm{~mW}$ in a $40 \mathrm{~nm} 3 \mathrm{~dB}$ bandwidth (cf. $200 \mu \mathrm{W}$ [1]).

(b) Use of new WDM couplers in our single source, single detector, dual-wavelength dual-Sagnac architecture. The theoretical loss of this Sagnac configuration is only $3 \mathrm{~dB}$ higher (due to spectral slicing of the source) than that of an ideal lossless Sagnac, and has only $12 \mathrm{~dB}$ minimum theoretical loss overall. This is $6 \mathrm{~dB}$ better than the $18 \mathrm{~dB}$ minimum calculated for the dual-path dual-Sagnac. The transfer functions of these new WDM's are much closer to the ideal "Top-Hat" functions, improving cross-talk by over $30 \mathrm{~dB}$, compared to 
the previous system. When averaged over the wavelength band of interest, they also give a lower insertion loss, vis. $0.8 \mathrm{~dB}$ as compared to a corresponding average of $1.4 \mathrm{~dB}$ for the fused taper WDM couplers.

(c) In the previously presented system, all signal processing was carried out off-line after storing measurement data. Our new system utilizes real-time data acquisition hardware to sample the sensor outputs before processing the results on-line. The computer can then be instructed either to, (i) automatically locate the three largest disturbances observed in the frequency domain or (ii) locate a disturbance of selected character, eg. of a specific frequency or amplitude. This system then calculates and displays the positions and the frequencies of each of the disturbances.

\section{METHOD OF OPERATION}

Our optical configuration is shown in Fig. 1. A broadband, low-coherence source is spectrally sliced into two wavelength bands, using WDM's, which then route the light along two essentially independent Sagnac interferometer loops. The first Sagnac loop is defined by the bi-directional path ABCEFHI, as read clockwise around the sensor. This circuit includes a fiber delay coil, C, a sensing fiber length, $E$, and $a$

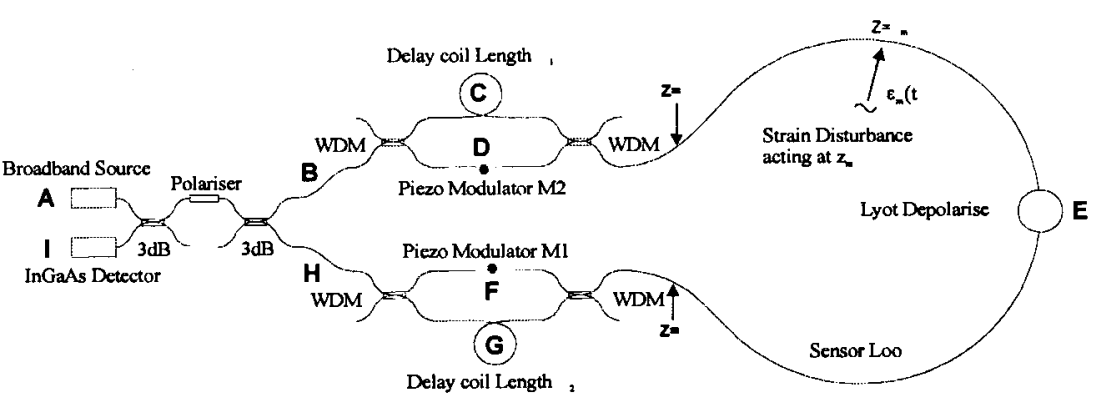

Fig. 1. Optical architecture of the dual-wavelength, dual-Sagnac interferometer. Two Sagnac interferometers are formed by spectral slicing and optical routing of a broadband superluminescent fiber source, such that each shares a common, offset section of the sensing loop. The signals generated by a time-varying strain disturbance are then observed for each of the interferometers by a common optical detector. piezoelectric phase modulator, F. Similarly, the second Sagnac interferometer is defined by routing light bi-directionally around a path labeled ABDEGHI. This again includes a piezoelectric modulator, $D$, a common sensing fiber length, $E$, and a fiber delay loop, G. The centers of each of the sensor loops are offset (in opposite directions) by half of the path length in the delay coils labeled $\mathrm{C}$ and $\mathrm{G}$, ensuring that, despite sharing a common fiber section, each Sagnac gives a different response to a common perturbation. This allows simultaneous evaluation of the position of the disturbance, its amplitude and its rate of change.

Each of the Sagnac's is phase-biased [9] with a sinusoidal strain signal The Sagnac bias signals are of different frequencies $\left(f_{1}\right.$ and $f_{2}$ respectively), each being one of a set of natural eigenfrequencies of the fiber loop. The bias frequencies $\left(f_{1}\right.$ and $f_{2}$ ) were chosen such that even their difference frequency $\left[f_{1}-f_{2}\right]$ was above the frequency range of the expected disturbance signals, (i.e. the base-bandwidth of the output of the sensor.) This phase bias allows both of the interferometers to share a common optical detector, as it provides amplitude-modulated carriers of a different frequency from each signal generated by the Sagnac. It also improves the sensitivity, by moving the interferometer's operating point to a high slope region in the sinusoidal phase response. Due to the non-linear nature of the Sagnac's phase response, when unperturbed, only even harmonics of the bias modulation are expected on the detected signal, (although, in practice, the detector will be band-limited to respond only up to the $2^{\text {nd }}$ harmonic). However, when a disturbance acts on the loop, odd harmonics of the bias frequency start to appear [9].

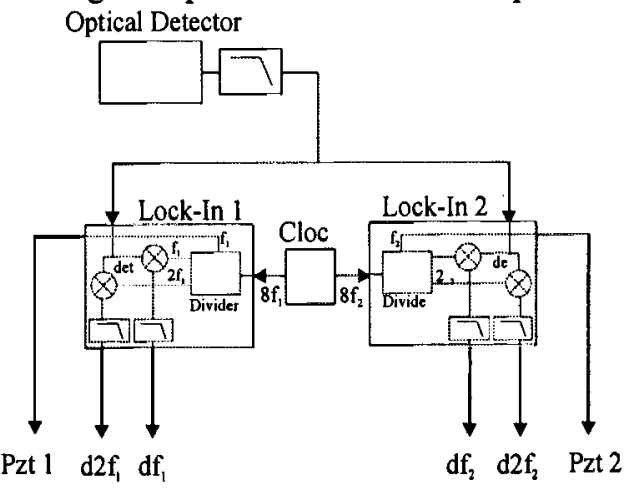

Fig. 2. Schematic of lock-in amplifier operation and piezoelectric drive signal synthesis.

After detection, the outputs from each of the Sagnacs are separated using a dual-active-homodyne electronic demodulation technique. Fig.2. shows the schematic of the dual lock-in amplifiers required to separate out the signals from each Sagnac. Each of the lock-in's monitor the components, $f_{1}, f_{2}, 2 f_{1}$, and $2 f_{2}$ respectively, of the bias frequencies in the detected signal, by multiplying the detected signal by the corresponding frequency before filtering the output. This generates four demodulated outputs $d f_{1}, d 2 f_{1}, d f_{2}$, and $d 2 f_{2}$, which may then be processed in the usual manner [1] to give a position-dependent result, independent of the rate of change or amplitude of the perturbation, assuming the system stays within a small signal approximation.

Unfortunately, for a real system, the situation is more complex. Due to non-ideal optical WDM components, there will inevitably be a certain amount of residual optical cross-talk, such that a further two optical paths become weakly allowed. These new paths are labeled 1) ABCEGHI and 2) ABDEFHI. The first allows light to travel around the sensor loop without incurring a phase bias modulation. This causes only a baseband 
disturbance signal to appear on the detector when the sensor loop is perturbed. Fortunately, the lock-in detection and post-filtering removes this component. The second cross-talk path is potentially more serious, as it allows light to be phase biased at frequencies corresponding to both Sagnacs. When these signals are demodulated, an error signal results in each, requiring a small correction to remove the effects. The analytical response of the system was fully modeled and cross-talk effects accounted for.

\section{EXPERIMENTAL}

\section{A. Description of optical apparatus}

This experimental arrangement is shown in Fig.1. A 980nm, 80mW laser diode (Lucent LUC263ENG) was used to single-end pump, a $25 \mathrm{~m}$ length of Er/Al-doped fiber (having a pump absorption of $5 \mathrm{dBm}^{-1}$ ) via a biconically fused fiber WDM (Etek 980/1550). The backward ASE provided a $4 \mathrm{~mW}$ source, having a center wavelength of $1555 \mathrm{~nm}$ and a $40 \mathrm{~nm}$ FWHM bandwidth. The polariser used was a Sifam SP15 device having a $25 \mathrm{~dB}$ extinction ratio. The $3 \mathrm{~dB}$ couplers used were standard biconically-tapered devices (Sifam SC155050/50). As previously stated, Dicon FWC-28.0 dichroic filter based WDM's were used, with transfer functions approaching (apart from $0.8 \mathrm{~dB}$ insertion loss) the ideal "Top-Hat" response. These had transmission in the band $1552-1565 \mathrm{~nm}$ and reflection between $1528-1544 \mathrm{~nm}$. The quoted optical cross-talk between channels in these WDM's was below $-10 \mathrm{~dB}$, giving a $-40 \mathrm{~dB}$ minimum cross-talk level for the system as a whole. The fiber lengths were measured as $\mathrm{L}=38.69 \mathrm{~km}$ (sensor loop) and $\mathrm{L}_{1}=3.55 \mathrm{~km}$ and $\mathrm{L}_{2}=4.05 \mathrm{~km}$ (delay coils) using an OTDR. The outputs from both Sagnacs were detected by a fiber-pigtailed gallium arsenide PIN detector (Nortel DPR2SB-43T2), having a $0.72 \mathrm{~A} / \mathrm{W}$ responsivity. Its output was amplified using a $512 \mathrm{kOhm}$ transimpedance amplifier, with a $3 \mathrm{~dB}$ bandwidth of $5 \mathrm{MHz}$, built in-house using a Burr Brown OPA655 Op-Amp. This detector output was later lowpass filtered, with a $3 \mathrm{~dB}$ frequency response of $120 \mathrm{kHz}$. This attenuated the $3 \mathrm{f}$ harmonics by approximately $40 \mathrm{~dB}$ compared to the $\mathrm{f}$ components. Polarization fading was minimized by incorporating an all-fiber LyotDepolarizer [11] into the sensor loop. This was constructed from $3 \mathrm{~m}$ and $6 \mathrm{~m}$ lengths of Hi-Bi $\left(\mathrm{l}_{\mathrm{b}}=2.93 \mathrm{~mm}\right)$ Panda fiber, joined with their polarization axis offset by $45^{\circ}$ relative to each other. To reduce effects from acoustic pick-up, the sensor loop fiber was wound onto six fiber drums and placed in an acoustically shielded box, with only a few short sections exposed to apply perturbations. Similarly, the optical components used to construct the interrogation optics were also placed into a separate acoustically shielded box.

\section{B. Positioning of a single tone disturbance.}

The system was set up as described and the excitation eigenfrequencies of each loop were chosen to be $40.59 \mathrm{kHz}$ and $50.73 \mathrm{kHz}$ respectively. The modulation depth of the sinusoidal phase bias was set to ensure operation at the system's quadrature point and maximize the demodulated output derived from the odd harmonics. A modulator to simulate disturbances was constructed using a loudspeaker as a driver. This deflected a beam of thin deformable plastic to which the fiber was attached. Simulated disturbances were then placed at several positions around the sensor loop, each giving a phase perturbation of approximately 0.1 radians amplitude, initially at a frequency of $125 \mathrm{~Hz}$. The four outputs from the lock-ins were sampled at $16 \mathrm{kHz}$ by a computer, which then performed an FFT operation on the data. Fig.3. shows an example of the observed frequency spectrum of the $f$ demodulated output showing a peak at $125 \mathrm{~Hz}$. The program was then instructed to locate this disturbance. It then performed a $4 \mathrm{~Hz}$ bandwidth filtering operation on all four lock-in channels, centered on $125 \mathrm{~Hz}$ for the $f$ demodulated channels and on DC for the $2 f$. The filtered outputs were then ratioed and the position calculated. Fig.4. shows the results from taking 100 averages of this disturbance at each point, with each point corresponding to different selected positions around the loop. As can be seen, the linearity of the system shows good agreement with theory.

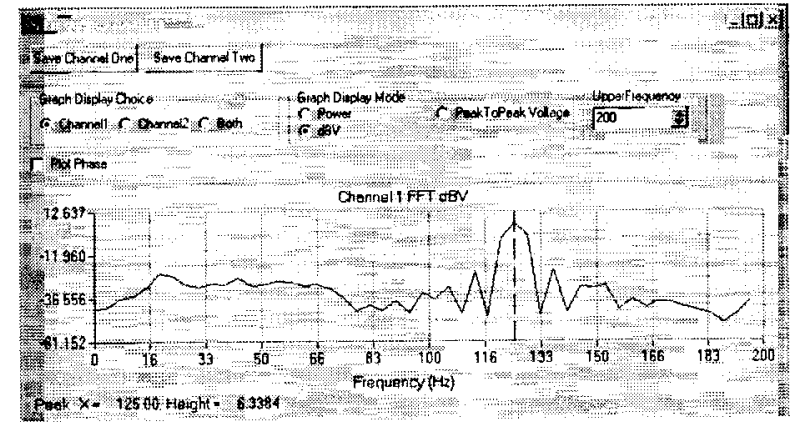

Fig. 3. Example of FFT Spectrum of $f$ demodulated signal for a Sagnac perturbed with a $125 \mathrm{~Hz}$ tone disturbance, 0.1 radians in amplitude.

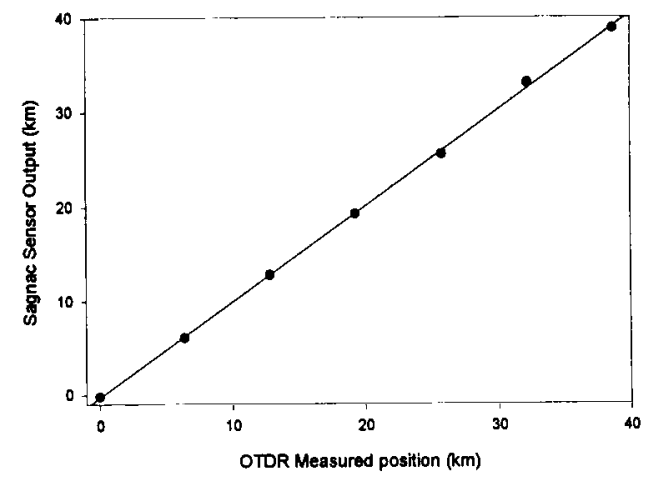

Fig. 4. Plotted results for 100 averages of a tone disturbance of $125 \mathrm{~Hz}$, producing a phase perturbation 0.1 radians in amplitude, acting at several positions around the sensing loop of our dual-Sagnac architecture. 


\section{Positioning of multiple disturbances}

We shall now describe the method for location of multiple disturbances. As previously stated, the computer signal processing program could firstly be instructed to automatically locate the three largest-amplitude disturbances acting on the loop. The system was then instructed to locate applied disturbances at frequencies of $110 \mathrm{~Hz}, 130 \mathrm{~Hz}$ and $150 \mathrm{~Hz}$, applied at $0 \mathrm{~km}$, $12.2 \mathrm{~km}$ and $32.2 \mathrm{~km}$ respectively. When "located" by using our new system, they were determined to lie at $0.14 \mathrm{~km}, 11.6 \mathrm{~km}$ and $33.65 \mathrm{~km}$. We believe the slight disagreement between observed and measured disturbance location is due to slight differences in the frequency responses of each of the lock-ins, not so far accounted for in the software. Future work will attempt to improve on this. Fig.5. shows a screen capture of the computer display, when positioning multiple disturbances at different frequencies.

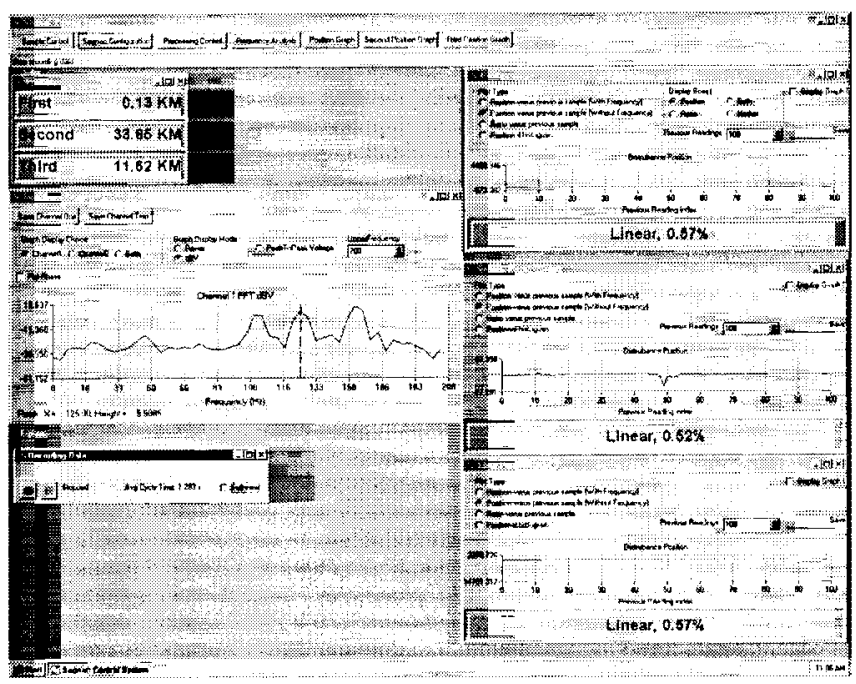

Fig 5. Screen capture of multiple disturbance positioning program for dualSagnac system

\section{CONCLUSIONS}

We have experimentally demonstrated a dual-wavelength dual-Sagnac optical sensor system with greatly improved location and discrimination capability. We have located 0.1 radian sinusoidally-varying phase disturbances acting on a $40 \mathrm{~km}$ long sensing loop with a $100 \mathrm{~m}$ positional sensitivity. Improved signal processing software has allowed real-time processing of results and for the first time it has permitted 3 disturbances, acting at different locations, to be simultaneously and separately positioned. The complications caused by optical crosstalk between the two Sagnacs has been fully modelled and used to calibrate the system. We believe that it should be possible, with further processing, to account for the non-linear nature of the system's response, allowing a wider range of disturbances to be positioned, reducing complications arising from background acoustic noise.

\section{REFERENCES}

[1] S.J. Russell, J.P. Dakin, "Location of time-varying strain disturbances over a $40 \mathrm{~km}$ fiber section, using a dual-Sagnac interferometer with a single source and detector." Proceeding OFS-13, Koyngju, Korea, SPIE Vol 3746, pp.580-583, April 1999.

[2] J.P. Dakin, D.A. Pearce, A.P. Strong, C.A. Wade, "A novel distributed optical fibre sensing system enabling location of disturbances in a Sagnac loop interferometer," Proceedings SPIE, vol 838, 1987.

[3] J.P. Dakin, D.A. Pearce, A.P. Strong, C.A. Wade, "A novel distributed optical fibre sensing system enabling location of disturbances in a Sagnac loop interferometer," Proceedings EFOC/LAN, pp276-279, 1988.

[4] S.J.Spammer, P.L. Swart. A. Boosen, "Interferometric distributed fiber optical sensor", Applied Optics, Vol 35, No 22, pp 4522-4523, 1996

[5] S.J. Spammer, A.A. Chtcherbakov, P.L. Swart, "Dual wavelength Sagnac-Michleson distributed optical fiber sensor", Proceedings SPIE pp 2838-2834, 1996.

[6] E.Ronnekleiv, K. Blotekjaer, K. Kranes, “Distributed fiber sensor for location of disturbances”, Proceedings OFS-9, PD7, 1993.

[7] X.J. Fang, "Fiber-optic distributed sensing by a two-loop Sagnac interferometer" Optical letters, vol 21, No 6, pp 444-446 1996.

[8] S.J. Spammer, A.A. Chtcherbakov, P.L. Swart, "Distributed dual wavelength Sagnac impact sensor", microwave and optical technology letters, vol 17, No 3, pp 170-173, 1998.

[9] A.Danbridge,A.B.Tveten,T.G.Giallorenzi, "Homodyne Demodulation Scheme for Fiber Optic Sensors Using Phase Generated Carrier", IEEE Transactions on microwave theory and techniques, vol MTT-30, No 10, pp 1635-1641, October 1982.

[10] N.W.McLachlan, "Bessel functions for engineers", Publisher Oxford University Press 1946.

[11] P.R.Cooper, "All fibre Lyot Depolarizer", Optics and laser technology, vol 18, No 2, April, pp 99-100 1986. 